\title{
Polyphenol- and fibre-rich dried fruits with green tea attenuate starch-derived postprandial blood glucose and insulin: a randomised, controlled, single-blind, cross-over intervention
}

\author{
H. Nyambe-Silavwe and G. Williamson* \\ School of Food Science and Nutrition, University of Leeds, Leeds LS2 9JT, UK \\ (Submitted 7 December 2015 - Final revision received 17 March 2016 - Accepted 9 May 2016 - First published online 9 June 2016)
}

\begin{abstract}
Polyphenol- and fibre-rich foods (PFRF) have the potential to affect postprandial glycaemic responses by reducing glucose absorption, and thus decreasing the glycaemic response of foods when consumed together. A randomised, single-blind, cross-over study was conducted on sixteen healthy volunteers to test whether PFRF could attenuate postprandial blood glucose in healthy volunteers when added to a source of carbohydrate (starch in bread). This is the first study to examine the effects of a meal comprised of components to inhibit each stage of the biochemical pathway, leading up to the appearance of glucose in the blood. The volunteers were fasted and attended four visits: two control visits (bread, water, balancing sugars) and two test visits (single and double dose of PFRF) where they consumed bread, water and PFRF. Blood samples were collected at 0 (fasted), 15, 30, 45, 60, 90, 120, 150 and 180 min after consumption. The PFRF components were tested for $\alpha$-amylase and $\alpha$-glucosidase inhibitory potential in vitro. Plasma glucose was lower after consumption of both doses compared with controls: lower dose, change in mean incremental areas under the glucose curves (IAUC) $=-27.4$ (sD 7.5) $\%, P<0 \cdot 001$; higher dose, IAUC $=-49 \cdot 0$ (sD 15.3$) \%, P<0.001$; insulin IAUC was also attenuated by -46.9 (sD 13.4) $\%, P<0 \cdot 01$. Consistent with this, the polyphenol components of the PFRF inhibited $\alpha$-amylase (green tea, strawberry, blackberry and blackcurrant) and $\alpha$-glucosidase (green tea) activities in vitro. The PFRF have a pronounced and significant lowering effect on postprandial blood glucose and insulin response in humans, due in part to inhibition of $\alpha$-amylase and $\alpha$-glucosidase, as well as glucose transport.
\end{abstract}

Key words: Postprandial glucose: Diabetes: $\alpha$-Amylase: $\alpha$-Glucosidase: Polyphenols: Fibre

Postprandial hyperglycaemia and high glycaemic index (GI) diets in humans play a major role in the development of type 2 diabetes $^{(1-4)}$, and furthermore low GI diets show favourable changes in health markers such as plasminogen activator inhibitor- $1^{(5)}$, glycated proteins ${ }^{(6,7)}$ and fasting blood glucose, especially in those with already elevated values ${ }^{(7)}$. Recent metaanalyses report that low-carbohydrate and low-GI diets have promising effects in diabetes management ${ }^{(8)}$. Strategies to reduce the GI of foods, even without altering the total carbohydrate content, are therefore of growing interest for reducing diabetes risk. The GI and response depend on several related factors including the nature and amount of carbohydrate, the rate of carbohydrate digestion in the gastrointestinal tract, the rate of absorption of the resulting glucose, the insulin response to the absorbed sugar and the intrinsic insulin sensitivity ${ }^{(9)}$. The presence of naturally occurring polyphenols has been associated with low GI foods for many decades ${ }^{(10)}$. Fibre can also play a role in reducing hyperglycaemia by delaying glucose absorption, increasing insulin secretion and sensitivity, and binding of bile acids $^{(11)}$. In addition, soluble fibre attenuates postprandial glucose by increasing the viscosity in the gastrointestinal tract, which disturbs carbohydrate breakdown and glucose absorption ${ }^{(12)}$. Possible mechanisms by which polyphenols may affect postprandial glycaemia are the inhibition of carbohydrate digesting enzymes and glucose transporters, stimulation of pancreatic $\beta$-cells to secrete insulin, activation of insulin receptors, modulation of the release of glucose from the liver and effects on intracellular signalling pathways and gene expression ${ }^{(13,14)}$. The potential action of polyphenols can be compared with that of acarbose, an $\alpha$-glucosidase and $\alpha$-amylase inhibitor, which reduces diabetes risk ${ }^{(15)}$. The Study To Prevent Non-Insulin dependent Diabetes Mellitus trial in impaired glucose tolerant (IGT) subjects showed a $36 \%$ risk reduction in the progression to diabetes after treatment with acarbose ${ }^{(16)}$. The use of diet-related intervention either on its own or in combination with acarbose would be an alternative to the use of acarbose alone, which can lead to side-effects such as flatulence, nausea and diarrhoea.

Some polyphenols inhibit starch-digesting enzymes ( $\alpha$-amylase and $\alpha$-glucosidase) in addition to the glucose transporters SGLT1 (SLC5A1) and GLUT2 (SLC2A2) ${ }^{(13)}$. Most intervention

Abbreviations: GI, glycaemic index; IAUC, incremental areas under the glucose curves; $\mathrm{IC}_{50}$, concentration required for $50 \%$ inhibition; PFRF, polyphenol- and fibre-rich food.

* Corresponding author: G. Williamson, email g.williamson@leeds.ac.uk 
studies carried out so far have focused on the effect of polyphenols with the endogenous carbohydrates already present in the food; however, addition of polyphenols and fibre to reduce the GI of that food has not been fully explored, but it is the normal way in which most foods are consumed - that is, as a total meal in combination with other foods. We therefore tested the hypothesis that a combination of components in the diet (polyphenols and fibre), capable of inhibiting the different stages of starch digestion, would reduce postprandial blood glucose and insulin using a randomised, controlled, singleblind, cross-over intervention. The test diet consisted of an $\alpha$-glucosidase inhibitor (green tea), $\alpha$-amylase inhibitors (green tea, blackberry, blackcurrant and strawberry) and glucose transport inhibitors (apple peel and strawberry), with all fruits providing fibre as well.

\section{Methods}

\section{Subjects}

The recruitment of subjects was carried out at the University of Leeds, School of Food Science and Nutrition, Leeds, UK. Poster advertisements around the University of Leeds notice boards were used to recruit interested potential volunteers, who were then screened for fasting blood glucose (required to be between 3.9 and $5.9 \mathrm{mmol} / \mathrm{l}$ ). They were then asked to assess themselves using criteria to ensure that they could be classified as healthy and free of symptomatic disease. The eligibility criteria were as follows: aged 18-75 years, apparently healthy, not diabetic, not on long-term prescribed medications, not pregnant or lactating and not on a special diet (e.g. for losing weight or fruit supplements). The preferred order of consumption was reference meal at the first and last visit, with randomised consumption of the high and low dose on their second and third visits. However, because of unavailability of some volunteers, who started late and required a break of 3 weeks after two visits, the order was changed for them to reference, high/low dose, reference, then low/high dose, in order to start with the control meal after a break (Fig. 1). In total, sixteen healthy volunteers aged 26 (SD 4) years with BMI of $24(\mathrm{sD} 3) \mathrm{kg} / \mathrm{m}^{2}$ gave their written informed consent and completed the four study visits as shown in Fig. 1. The fasting plasma glucose and insulin concentrations were 4.8 (sD 0.4 ) $\mathrm{mmol} / \mathrm{l}$ and 24 (SD 10) $\mathrm{pmol} / \mathrm{l}$, respectively.

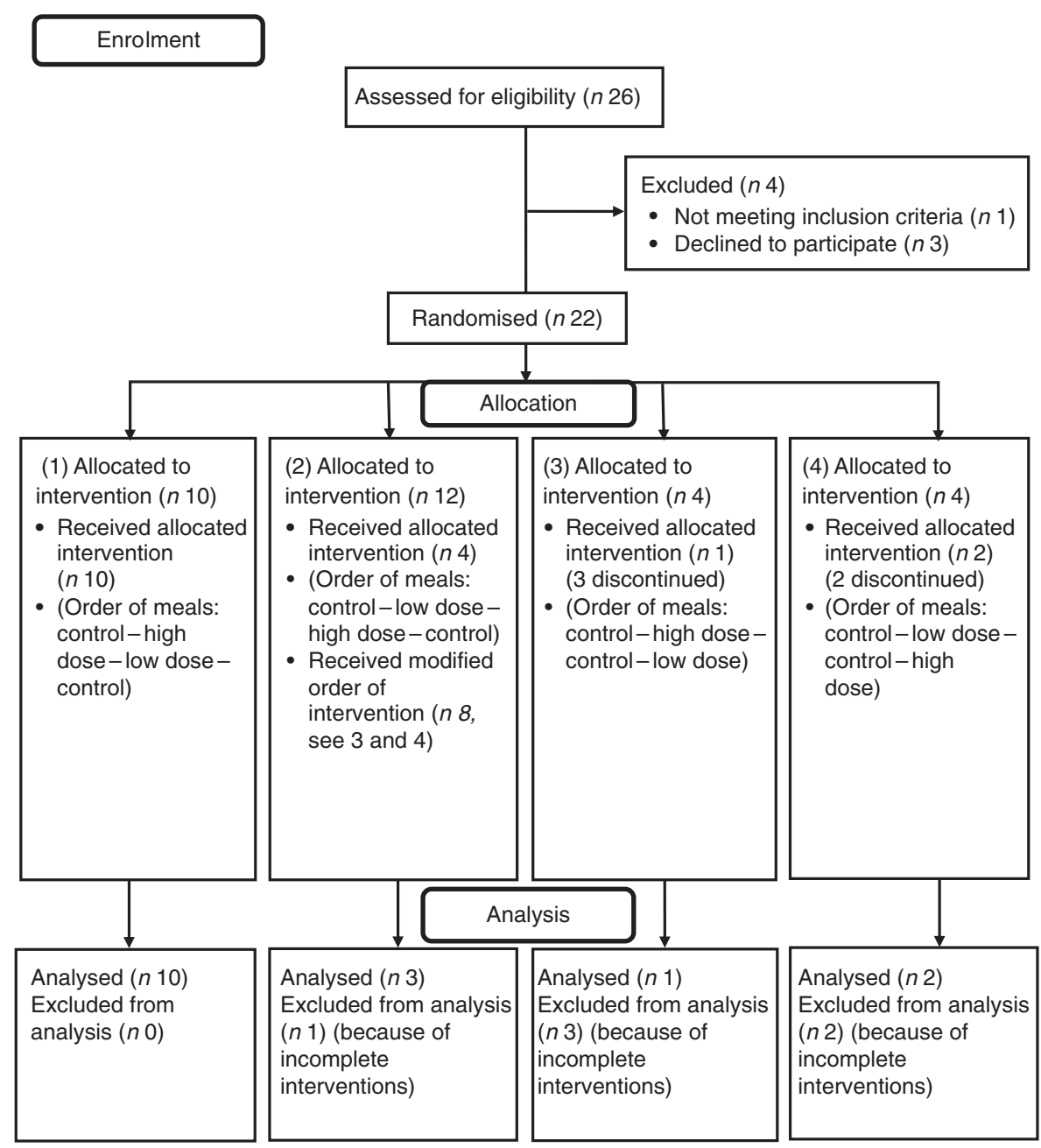

Fig. 1. Participant flow diagram. Block randomisation was used to generate the allocated sequences, which were assigned to participant codes. Sequences were automatically allocated to participants according to the participant codes. 


\section{Study design}

A randomised, controlled, single-blind, cross-over intervention was carried out on a total of sixteen healthy volunteers with the primary outcome of postprandial blood glucose AUC. Because of the nature of the test meals, it was impossible to blind participants. However, analysis of the plasma samples was blinded and was only unblinded after data analysis. Subjects were cannulated to ensure comfortable collection of blood samples. Each participant had four visits, two of which were reference meals and two visits were test meals (single and double dose of polyphenol- and fibre-rich food (PFRF) in a randomised pattern).

\section{Test meals}

All meals contained 109.0 (SD 1.2) g of white bread (50 g available carbohydrate as analysed by the method of Englyst $\left.{ }^{(17)}\right)$. The higher dose consisted of $1 \mathrm{~g}$ green tea powder in $200 \mathrm{ml}$ water, with $20 \mathrm{~g}$ each of apple peel, blackberry, blackcurrant and strawberry freeze-dried powders mixed with water to make a paste and spread on the bread. The reference meal included $0 \cdot 8,5.4$ and $8.6 \mathrm{~g}$ of sucrose, glucose and fructose, respectively, dissolved in $200 \mathrm{ml}$ water to standardise the amounts of sugars present in the extracts of the high dose. The volunteers consumed the reference meal on two of the visits to determine any variability in the measurements ${ }^{(18)}$. The lower dose of the test meal contained half the amount of fruits and green tea with half the amounts of balancing sugars dissolved in $200 \mathrm{ml}$ water to equalise the amount of sugars present in all doses. A PFRF containing polyphenols that are effective inhibitors of different stages of starch digestion and absorption was used in this study. It comprised of an $\alpha$-glucosidase inhibitor (green tea) ${ }^{(19-21)}$, $\alpha$-amylase inhibitors (green tea, blackberry, blackcurrant and strawberry) ${ }^{(22-24)}$ and glucose transport inhibitors (apple peel and strawberry $)^{(25-27)}$, with all fruits providing some fibre. The PFRF components were analysed for total polyphenol content, specific major polyphenols and for $\alpha$-amylase and $\alpha$-glucosidase inhibition in vitro.

\section{Materials}

Human salivary amylase, acetone extract of rat intestine, sucrose, maltose, glucose, fructose and glucose hexokinase reagent were from Sigma-Aldrich Co. Ltd. Freeze-dried fruit extracts were from Healthy supplies, UK, and green tea powder was from Nestlé Research Centre. The insulin immunoassay kit was from Mercodia AB.

\section{Study protocol}

The University of Leeds, Faculty of MaPS and Engineering Ethics Committee (MEEC) approved the study protocol (MEEC 12-037), and the protocol was registered with ClinicalTrials.gov, ID number NCT01994135. Each participant had one visit per week, and hence completed the study in four weeks, with body weight and height measurements taken on the first visit. On each visit, a cannula was inserted in the forearm of each subject.
A fasting blood sample was collected, and after that the volunteer consumed the meal; the timer started upon first bite or sip. The volunteers consumed the whole meal, and blood samples were collected after 15, 30, 45, 60, 90, 120, 150 and $180 \mathrm{~min}$. Neither harm nor side-effects occurred during the consumption of the meals. Blood samples were collected in fluoride/oxalate and EDTA tubes for glucose and insulin measurements, respectively, and immediately placed on ice. The tubes were then centrifuged within $15 \mathrm{~min}$ at $4000 \mathrm{~g}$ at $4^{\circ} \mathrm{C}$ for $15 \mathrm{~min}$. Thereafter, plasma samples were placed in storage tubes and stored at $-80^{\circ} \mathrm{C}$. Plasma glucose concentrations were determined using hexokinase linked to NADH oxidation (Sigma-Aldrich) and insulin concentrations by immunoassay.

\section{Statistical analysis}

The incremental areas under the glucose curves (IAUC) were calculated for each subject for each visit using the trapezoidal rule. Data were analysed using the two-tailed paired $t$ test analysis, and the results were confirmed using the one-factor repeated-measures ANOVA between the two references, reference and dose 1 , reference and dose 2 and between lower and higher doses. Sample size was determined by designing the trial to have $90 \%$ power to detect a clinical difference of $15 \%$ IAUC between test and reference meals. The study required fifteen participants each for reference and test meals. With participants being controls of themselves, a minimum of fifteen participants was required.

\section{Enzyme assays in vitro}

Green tea and fruit extracts were tested separately in vitro to determine the inhibition of starch-digesting enzymes. Measurement of human salivary $\alpha$-amylase inhibition was carried out as described previously ${ }^{(28)}$. In brief, sugars were removed from the fruit extracts using oasis max 3-cc cartridges. The sugar-free extracts, in water, were used as the inhibitor stock for the experiments. The 500- $\mu$ l assay volume consisted of $200 \mu \mathrm{l}$ of amylose or amylopectin, $50 \mu \mathrm{l}$ of PBS and $50 \mu \mathrm{l}$ of the inhibitor, and the assay was started by adding $200 \mu \mathrm{l}$ of $1.25 \mathrm{U} / \mathrm{ml}$ human salivary $\alpha$-amylase (Sigma-Aldrich Co. Ltd). After $10 \mathrm{~min}$ of incubation at $37^{\circ} \mathrm{C}$, the reaction was stopped by placing the tubes in a water bath at $100^{\circ} \mathrm{C}$. The tubes were cooled to room temperature, and solid-phase extraction (SPE) was used to remove polyphenols from the assay contents before the addition of $1 \mathrm{ml} \mathrm{3,5-dinitrosalicylic} \mathrm{acid} \mathrm{(DNS),} \mathrm{as}$ some polyphenols can interact with DNS, and thus interfere with the reaction ${ }^{(28)}$. A plate reader was used to measure the absorbance at $540 \mathrm{~nm}$, and inhibition was calculated as a percentage of the control.

The inhibition of rat $\alpha$-glucosidase method was adapted from Adisakwattana et al. $^{(29)}$. The apparent $K_{m}$ values for sucrose, iso-maltose and maltose were determined and calculated using the Lineweaver-Burk plot with a chosen enzyme concentration and incubation times giving linear rates of reaction. The $K_{m}$ values obtained were 16, 6 and $3 \mathrm{~mm}$ for sucrose, iso-maltose and maltose, respectively, and these were subsequently used as the substrate concentrations in the assays. 
The assay consisted of substrate ( $200 \mu \mathrm{l}$ of sucrose, iso-maltose or maltose), sodium phosphate buffer $(50 \mu \mathrm{l}, 10 \mathrm{~mm})$, inhibitor or extra buffer $(50 \mu \mathrm{l})$, and the reaction was started by adding $200 \mu \mathrm{l}$ of acetone-derived protein intestinal extract from rat intestine $(20 \mathrm{mg} \mathrm{solid} / \mathrm{ml}$ for sucrose and iso-maltose and $4 \mathrm{mg}$ solid $/ \mathrm{ml}$ for maltose). After incubation at $37^{\circ} \mathrm{C}$ for $20 \mathrm{~min}$, the reaction was stopped by placing the tubes in a water bath at $100^{\circ} \mathrm{C}$ for $10 \mathrm{~min}$. After cooling to room temperature, SPE was used to remove polyphenols, and the resulting solution was analysed for glucose at $340 \mathrm{~nm}$ in a plate reader using hexokinase, which catalyses $\mathrm{NADH}$ reduction. Inhibition was calculated as a percentage of the control.

\section{Total polyphenols by Folin's assay}

Extracts from the fruits were analysed for total polyphenols using Folin's assay ${ }^{(30)}$, including a control for each sample to account for any interference from, for example, ascorbic acid, and data were expressed as $\mu \mathrm{g} / \mathrm{mg}$ gallic acid equivalents. To 15- $\mathrm{ml}$ falcon tubes, $1 \mathrm{ml}$ of each solution (standards and samples) was added. The assay was conducted by adding $5 \mathrm{ml}$ of the Folin-Ciocalteu reagent to all the samples and standards. The tubes were capped, votexed and $4 \mathrm{ml}$ of sodium carbonate solution was added within 3-8 min from the addition of the Folin-Ciocalteu reagent. The tubes were capped, votexed quickly and then placed in the water bath and incubated at $26^{\circ} \mathrm{C}$ for $2 \mathrm{~h}$. Absorbance readings at $765 \mathrm{~nm}$ were relative to a gallic acid standard curve.

\section{Analysis of polyphenols by HPLC}

The major polyphenols in the fruit samples and green tea were characterised using HPLC as described previously ${ }^{(27)}$. An Agilent 1200 SL system (Agilent Technologies) equipped with a diode array detector (DAD) was used. It comprised of a binary pump, degasser, column oven $\left(35^{\circ} \mathrm{C}\right)$ and a well plate autosampler $\left(5^{\circ} \mathrm{C}\right)$. A Zorbax Eclipse plus C18 column $(1.8 \mu \mathrm{m}$, $100 \times 2.1 \mathrm{~mm})$ and Agilent-Zorbax eclipse XDB-C18 $(1.8 \mu \mathrm{m}$, $50 \times 4.6 \mathrm{~mm}$ ), both from Agilent Technologies, were used for green tea and fruit extracts, respectively. Other parameters were 5 - $\mu$ injection volume, at $0.5 \mathrm{ml} / \mathrm{min}$ flow rate with needle wash in the flush point for $3 \mathrm{~s}$. For all analyses, ultrapure, nucleasefree water $\left(\geq 18.2 \mathrm{M} \Omega \mathrm{cm}\right.$ at $\left.25^{\circ} \mathrm{C}\right)$ from a Millipore $\mathrm{Q}$ water purifying system (Millipore) was used. For sample preparation, a Genevac (EZ-2 plus model, Fisher Scientific Ltd) was used for centrifugal evaporation. Polyphenols were identified by their retention times compared with authentic standards, and standard curves were used for quantification.

\section{Sugar analysis by HPLC}

Sugar quantification of the fruit extracts was conducted using a Shimadzu HPLC instrument equipped with a model DGU-20 A5 degasser, a LC-20 AD XR pump system, a SIL-20 AC XR auto sampler (Shimadzu), column oven, a DAD system (SPD-M20A; Shimadzu) and a Shimadzu ELSD-LTII low-temperature, evaporative, light-scattering detector. A sample volume of $10 \mu \mathrm{l}$ was injected, and separations were achieved on a Prevail Carbohydrate ES $5-\mu \mathrm{m}$ column $(250 \times 4.6 \mathrm{~mm}$; Grace $)$. The column was held at $20^{\circ} \mathrm{C}$, and individual sugars were eluted isocratically using a $1 \mathrm{ml} / \mathrm{min}$ flow of $75 \%$ acetonitrile. Solutions of standard sugars prepared in water (Millipore, HPLC grade) with concentrations between 0 and $10 \mathrm{mg} / \mathrm{ml}$ were used for the calibration curve. The sugars were identified by their retention time characteristics at $40^{\circ} \mathrm{C}$. Quantification was achieved using standard calibration curves obtained by plotting area $v$. concentration $\left(r^{2}>0.98\right)$. Data from the sugar analysis allowed balancing of glucose, fructose and sucrose in the fruit in the control samples as indicated above.

\section{Fibre estimation}

The AOAC (Association of Official Analytical Chemists) $\operatorname{method}^{(31)}$ was used for fibre determination by Healthy Supplies.

\section{Results}

\section{Polyphenol and sugar analysis}

Total polyphenol contents of the fruits and green tea are shown in Table 1, and all data fell within the normal range as recorded in phenol explorer ${ }^{(32)}$. Sugar analysis of PFRF gave a total of $0 \cdot 8,5 \cdot 4$ and $8.6 \mathrm{~g} / 80 \mathrm{~g}$ sucrose, glucose and fructose, respectively, and

Table 1. Polyphenol content of green tea and freeze-dried fruits* (Mean values and standard deviations; $n$ 3)

\begin{tabular}{|c|c|c|c|c|c|}
\hline \multirow[b]{2}{*}{ Extracts } & \multicolumn{2}{|c|}{$\begin{array}{l}\text { Total polyphenols ( } \mu \mathrm{g} / \mathrm{mg} \text { GAE) } \\
\text { fresh weight basis }\end{array}$} & \multirow[b]{2}{*}{ Specific polyphenols by HPLC } & \multirow[b]{2}{*}{ Mean $(\mathrm{mg} / \mathrm{g})$} & \multirow[b]{2}{*}{ SD } \\
\hline & Mean & SD & & & \\
\hline \multirow[t]{4}{*}{ Green tea } & 541 & 25 & $(-)$-Epigallocatechin gallate & $199 \cdot 8$ & $6 \cdot 7$ \\
\hline & & & (-)-Epigallocatechin & 124.4 & $9 \cdot 3$ \\
\hline & & & (-)-Epicatechin gallate & 34.4 & 1.9 \\
\hline & & & (-)-Epicatechin & 23.3 & $2 \cdot 4$ \\
\hline \multirow{2}{*}{ Apple peel } & 217 & 3 & Phlorizin & 1.82 & 0.03 \\
\hline & & & Quercetin-3-O-rhamnoside & $1 \cdot 13$ & 0.02 \\
\hline Blackberry & 295 & 3 & Cyanidin-3-O-glucoside & 7.01 & 0.08 \\
\hline Blackcurrant & 303 & 0 & Cyanidin-3-O-rutinoside & 1.04 & 0.03 \\
\hline Strawberry & 315 & 2 & Pelargonidin-3-O-glucoside & 4.5 & 0.1 \\
\hline
\end{tabular}

GAE, gallic acid equivalents.

* Total polyphenol contents and specific polyphenol contents of green tea and extracts from the tested fruit as analysed by Folin's assay and HPLC. 
these values were used to balance the control meal. Fibre contents were $0.22,0.53,0.43$ and $0.2 \mathrm{~g} / 100 \mathrm{~g}$ dry weight in apple peel, blackberry, blackcurrant and strawberry, respectively.

\section{Postprandial plasma glucose and insulin}

Both the low-dose and the high-dose test meals containing PFRF showed a significant dose-dependent decrease in the mean glucose IAUC compared with the control meals (Fig. 2), $-27.4(\mathrm{sD} 7.52) \%(P<0.01)$ and $-49 \cdot 0(\mathrm{SD} 15.3) \%(P<0 \cdot 01)$, respectively, with no significant difference between the two reference (control) meals. The peak glucose concentration was also significantly lower in both PFRF test meals compared with the reference meals. There was a reduction in insulin IAUC for the PFRF meal compared with the reference meal of -46.9 (SD 13.4)\% $(P<0.01)$ (Fig. 3). The PFRF meal also attenuated the peak postprandial insulin concentration, but any differences had disappeared by about $120 \mathrm{~min}$.

\section{Inhibition of $\alpha$-amylase and $\alpha$-glucosidase activities}

Green tea and extracts from blackberry, blackcurrant and strawberry inhibited human salivary $\alpha$-amylase $\left(\mathrm{IC}_{50}\right.$ values $=$ $0.009,1.2,1.5$ and $2.5 \mathrm{mg}$ dry powder/ml water (amylose as substrate); 0.025, 1.6, 1.7 and $3.9 \mathrm{mg} / \mathrm{ml}$ (amylopectin as substrate)) (Fig. 4 and Table 2), where $\mathrm{IC}_{50}$ is the concentration required for $50 \%$ inhibition. Green tea inhibited maltase, sucrase and iso-maltase in vitro with $\mathrm{IC}_{50}$ values of $0 \cdot 02,2 \cdot 3$ and $2 \cdot 0 \mathrm{mg} \mathrm{solid} / \mathrm{ml}$ water, respectively (Table 2 and Fig. 4(c)).

\section{Discussion}

Consumption of foods rich in polyphenols and fibre (PFRF) together with bread resulted in a highly significant dose-dependent lowering of the glucose AUC, as well as an associated attenuation of insulin. Although there was substantial inter-individual variation, the cross-over design minimised the consequences of this, and the two curves obtained for the (a)

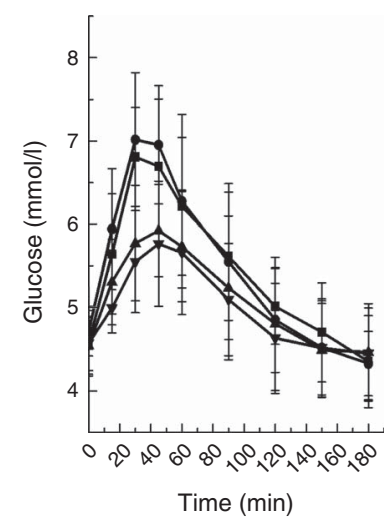

(b)

(c)

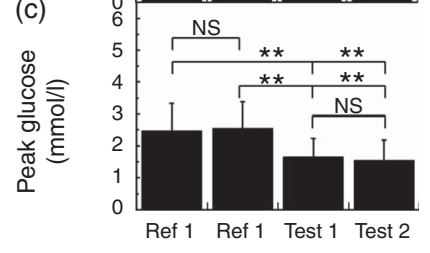

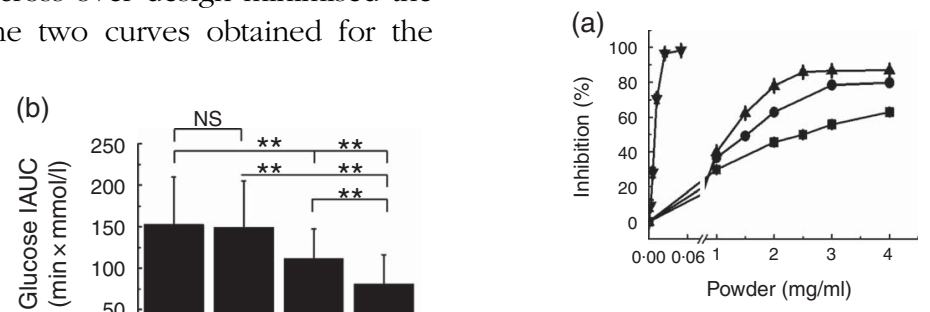

Fig. 2. Average glucose curves (a) after consumption of reference, test meal dose 1 and test meal dose 2 for sixteen volunteers. There is a significant difference between incremental areas under the glucose curves (IAUC) of reference meals and test meals (b) as well as between the peak rise in glucose concentration (c). ${ }^{* *} P<0.01$, NS denotes no significant difference. control meals were not significantly different. We propose that the effects of the test meals are most likely due to the results observed in the in vitro inhibition of human salivary $\alpha$-amylase (mainly green tea, blackberry, blackcurrant and strawberry), $\alpha$-glucosidase (green tea) and glucose transport (green tea, apple and strawberry) ${ }^{(26,27)}$, as well as the additional effect of fibre ${ }^{(33,34)}$. Although it is not possible to define the exact contribution of inhibition of the different steps (inhibition of $\alpha$-amylase, $\alpha$-glucosidase or glucose transport) to the attenuation of blood glucose, we would speculate that partial inhibition of multiple steps is important for the observed effect on the glycaemic response. These reductions can play a major, long-term role in the management or risk reduction of diabetes type 2, comparable with the drug acarbose, an $\alpha$-glucosidase inhibitor $^{(15)}$, as high concentrations of postprandial glucose lead (a)

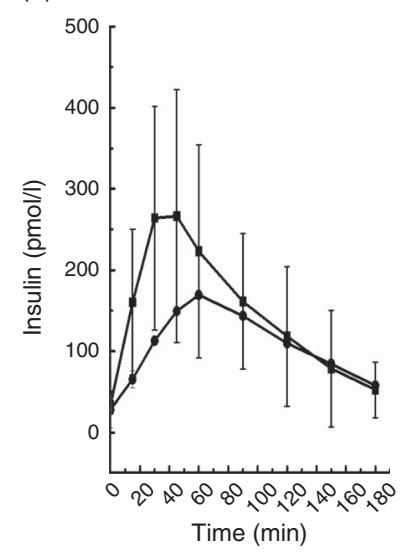

(b)

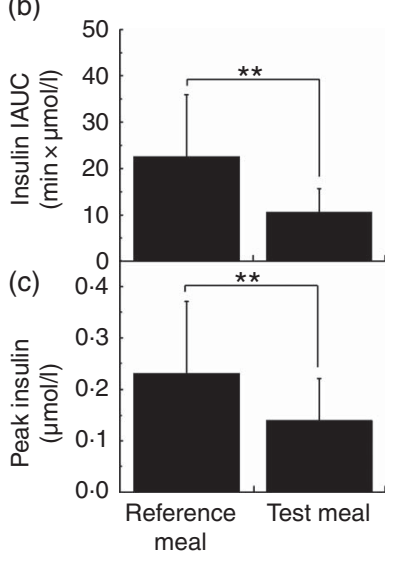

Fig. 3. Average insulin curves (a) after consumption of the reference meal and test meal dose 2 and for sixteen volunteers. There is a significance difference between insulin incremental areas under the glucose curves (IAUC) of the reference meal and the test meal (b) as well as between the peak rise in glucose concentration (c). ${ }^{\star \star} P<0.01$.
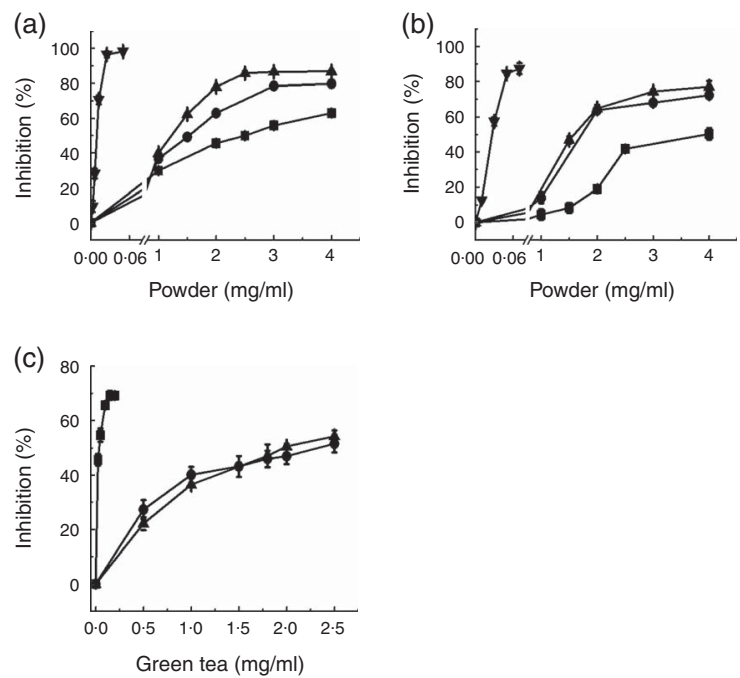

Fig. 4. Inhibition of human salivary $a$-amylase by green tea $(\boldsymbol{\nabla})$, freeze-dried strawberry $(\boldsymbol{\square})$, blackcurrant $(\boldsymbol{\bullet})$ and blackberry $(\boldsymbol{\Delta})$ using amylose as substrate (a) or amylopectin as substrate (b) and inhibition of maltase ( $\square$ ), sucrase $(\boldsymbol{\Lambda})$ and iso-maltase $(\boldsymbol{\bullet})$ by green tea (c). 
Table 2. Inhibition of carbohydrate-digesting enzymes

(Mean values and standard deviations)

\begin{tabular}{|c|c|c|c|c|c|c|c|c|}
\hline \multirow[b]{3}{*}{ Enzymes } & \multicolumn{8}{|c|}{$\mathrm{IC}_{50}(\mathrm{mg} / \mathrm{ml} \text { powder })^{\star}$} \\
\hline & \multicolumn{2}{|c|}{ Green tea } & \multicolumn{2}{|c|}{ Blackberry } & \multicolumn{2}{|c|}{ Blackcurrant } & \multicolumn{2}{|c|}{ Strawberry } \\
\hline & Mean & SD & Mean & SD & Mean & SD & Mean & SD \\
\hline Amylase (amylose) & 0.009 & 0.001 & $1 \cdot 22$ & 0.02 & 1.5 & 0.1 & $2 \cdot 47$ & 0.31 \\
\hline Amylase (amylopectin) & 0.025 & 0.001 & 1.57 & 0.21 & $1 \cdot 7$ & 0.1 & 3.85 & 0.05 \\
\hline Maltase & 0.02 & 0.01 & $>4$ & & $>4$ & & $>4$ & \\
\hline Iso-maltase & 2.02 & 0.01 & $>4$ & & $>4$ & & $>4$ & \\
\hline Sucrase & $2 \cdot 31$ & 0.02 & $>4$ & & $>4$ & & $>4$ & \\
\hline
\end{tabular}

* Experimental $\mathrm{IC}_{50}$ values for human salivary $a$-amylase using amylose and amylopectin as substrates and $\alpha$-glucosidase using maltose, sucrose and iso-maltose as substrates for green tea and freeze-dried fruits $(n 3)$.

to insulin resistance, pancreatic exhaustion, glucose intolerance and an increased insulin demand ${ }^{(35)}$. A highly significant effect on blood glucose was observed at both doses, with the higher dose (approximately 2-fold higher than the lower dose) leading to a doubling of the measured reduction in AUC. On the other hand, the peak glucose concentration was not further decreased by the higher dose.

A limited number of studies have reported the effects of isolated polyphenols or polyphenol-containing foods or extracts on postprandial glycaemia ${ }^{(36-49)}$, but the results are mixed with only some studies reporting significant differences between test meals and reference meals, and sometimes only at one or two time points, possibly owing to the use of different sugar sources - for example, glucose $\mathrm{e}^{(36,41,42,44-46)}$ or sucrose $^{(47)}$. A limited number of starch-based interventions, using rice, pancakes and white bread, have shown mixed results ${ }^{(38-40,43,48)}$. All of these abovementioned studies have not designed the study meal by considering the mechanism and including foods capable of attenuating the rate of each step of the digestive process. No significant difference was observed when a starch-based meal (pancakes) was used together with $100 \mathrm{~g}$ berries as the source of polyphenols ${ }^{(38)}$. When used alone, green tea as the sole source of polyphenols also did not give a significant difference in the IAUC $^{(43)}$. There was a significant difference in the IAUC when apple juice was used as a polyphenol source, clearly attributed to the inhibition of glucose transport by polyphenols in apple, especially phlorizin ${ }^{(41)}$. Polyphenols and fibre $(14.7 \mathrm{~g})^{(44)}$, present in lingonberries, nulled the glycaemic effect of the endogenous sugars present in the lingonberries. In vitro, polyphenols, phenolic acids and tannins in strawberry and apple reduced glucose transport using Caco-2 intestinal cell monolayers by inhibiting the glucose transporters SGLT1 and GLUT2. Phlorizin contributed $52 \%\left(\mathrm{IC}_{50}=146 \mu \mathrm{m}\right)$ and pelargonidin-3-O-glucoside $\left(\mathrm{IC}_{50}=802 \mu \mathrm{M}\right) 26 \%$ to the total inhibition by apple and strawberry, respectively ${ }^{(27)}$. These concentrations, together with those obtained for $\alpha$-amylase and $\alpha$-glucosidase inhibition (Table 2), were theoretically obtained in the gut lumen (Table 1) after taking into consideration calculated 3-fold dilution of consumed substances $^{(14)}$. For example, the $\mathrm{IC}_{50}$ value for $\alpha$-amylase inhibition by green tea was $0.009 \mathrm{mg} / \mathrm{ml}$ in vitro; 3-fold dilution in vivo would require $0.027 \mathrm{mg} / \mathrm{ml}$ in the original sample, and the test meals contained 2.5 and $5 \mathrm{mg} / \mathrm{ml}$ in the low and high dose, respectively. Therefore, we propose that polyphenols and fibre present in PFRF act together by inhibiting $\alpha$-amylase, $\alpha$-glucosidase and glucose transporters and leading to the observed reduced glycaemic response in vivo. The observed reduction in postprandial blood glucose and insulin can play a major role in the management and reducing the risk of type 2 diabetes, as hyperglycaemia is a risk factor for developing insulin resistance, IGT and consequently type 2 diabetes.

\section{Conclusion}

Polyphenols and fibre present in fruits, together with a cup of green tea, have a pronounced lowering effect on postprandial glucose and insulin when consumed together with a starchy food (bread), owing to the inhibition of different stages of starch digestion.

\section{Acknowledgements}

The authors thank the volunteers, Penny Rice (research nurse), David Tembo (assistance with sugar analysis) and the School of Food Science and Nutrition (University of Leeds) for meeting the research nurses' costs.

H. N.-S. received a student grant from the Commonwealth Scholarship Commission UK (ZMSC-2012-593) and the National Institute for Scientific and Industrial Research, Zambia, for $\mathrm{PhD}$ funding. Neither body was involved in any way in the design, interpretation or writing of the article.

H. N.-S.: design of study, carried out study and in vitro work, data analysis, writing the paper. G. W.: design of study, data analysis, writing the paper.

H. N.-S. has no conflicts of interest. This work did not receive funding from a commercial organisation, but G. W. has recently, or currently, received other research funding from Nestlé and Florida Department of Citrus, and conducted consultancy for Nutrilite, USA, and Suntory, UK.

\section{References}

1. Hodge AM, English DR, O'Dea K, et al. (2004) Glycemic index and dietary fiber and the risk of type 2 diabetes. Diabetes Care 27, 2701-2706.

2. McKeown NM, Meigs JB, Liu S, et al. (2004) Carbohydrate nutrition, insulin resistance, and the prevalence of the 
metabolic syndrome in the Framingham Offspring Cohort. Diabetes Care 27, 538-546.

3. Salmeron J, Manson JE, Stampfer MJ, et al. (1997) Dietary fiber, glycemic load, and risk of non-insulin-dependent diabetes mellitus in women. JAMA 277, 472-477.

4. Steven J, Ahn K, Juhaer I, et al. (2002) Dietary fiber intake and glycemic index and incidence of diabetes in African-American and white adults. Diabetes Care 25, 1715-1721.

5. Järvi AE, Karlström BE, Granfeldt YE, et al. (1999) Improved glycemic control and lipid profile and normalized fibrinolytic activity on a low-glycemic index diet in type 2 diabetic patients. Diabetes Care 22, 10-18.

6. Brand-Miller J, Hayne S, Petocz P, et al. (2003) Low-glycemic index diets in the management of diabetes a meta-analysis of randomized controlled trials. Diabetes Care 26, 2261-2267.

7. Livesey G, Taylor R, Hulshof T, et al. (2008) Glycemic response and health - a systematic review and meta-analysis: relations between dietary glycemic properties and health outcomes. Am J Clin Nutr 87, 258S-268S.

8. Ajala O, English P \& Pinkney J (2013) Systematic review and meta-analysis of different dietary approaches to the management of type 2 diabetes. Am J Clin Nutr 97, 505-516.

9. Augustin L, Franceschi S, Jenkins D, et al. (2002) Glycemic index in chronic disease: a review. Eur J Clin Nutr $\mathbf{5 6}$ 1049-1071.

10. Thompson LU \& Yoon JH (1984) Starch digestibility as affected by polyphenols and phytic acid. J Food Sci 49, 1228-1229.

11. Scazzina F, Siebenhandl-Ehn S \& Pellegrini N (2013) The effect of dietary fibre on reducing the glycaemic index of bread. Br J Nutr 109, 1163-1174.

12. Dikeman CL \& Fahey GC Jr (2006) Viscosity as related to dietary fiber: a review. Crit Rev Food Sci Nutr 46, 649-663.

13. Hanhineva K, Torronen R, Bondia-Pons I, et al. (2010) Impact of dietary polyphenols on carbohydrate metabolism. Int J Mol Sci 11, 1365-1402.

14. Williamson $G$ (2013) Possible effects of dietary polyphenols on sugar absorption and digestion. Mol Nutr Food Res 57, $48-57$.

15. Hanefeld M, Schaper F \& Koehler C (2008) Effect of acarbose on vascular disease in patients with abnormal glucose tolerance. Cardiovas Drugs Ther 22, 225-231.

16. Chiasson J-L (2006) Acarbose for the prevention of diabetes, hypertension, and cardiovascular disease in subjects with impaired glucose tolerance: the Study to Prevent Non-InsulinDependent Diabetes Mellitus (STOP-NIDDM) Trial. Endocr Pract 12, 25-30.

17. Englyst HN, Kingman SM \& Cummings JH (1992) Classification and measurement of nutritionally important starch fractions. Eur J Clin Nutr 46, S33-S50.

18. Brouns F, Bjorck I, Frayn KN, et al. (2005) Glycaemic index methodology. Nutr Res Rev 18, 145-171.

19. Honda M \& Hara Y (1993) Inhibition of rat small intestinal sucrase and $\alpha$-glucosidase activities by tea polyphenols. Biosci Biotechnol Biochem 57, 123-124.

20. Kamiyama O, Sanae F, Ikeda K, et al. (2010) In vitro inhibition of alpha-glucosidases and glycogen phosphorylase by catechin gallates in green tea. Food Chem 122, 1061-1066.

21. Matsui T, Tanaka T, Tamura S, et al. (2007) alpha-Glucosidase inhibitory profile of catechins and theaflavins. I Agric Food Chem 55, 99-105.

22. Forester SC, Gu Y \& Lambert JD (2012) Inhibition of starch digestion by the green tea polyphenol, (-)-epigallocatechin3-gallate. Mol Nutr Food Res 56, 1647-1654.

23. McDougall GJ, Shpiro F, Dobson P, et al. (2005) Different polyphenolic components of soft fruits inhibit alpha-amylase and alpha-glucosidase. J Agric Food Chem $\mathbf{5 3}$ 2760-2766.

24. McDougall GJ \& Stewart D (2005) The inhibitory effects of berry polyphenols on digestive enzymes. Biofactors $\mathbf{2 3}$, 189-195.

25. Hossain SJ, Kato H, Aoshima H, et al. (2002) Polyphenolinduced inhibition of the response of $\mathrm{Na}+$ /glucose cotransporter expressed in Xenopus oocytes. J Agric Food Chem 50, 5215-5219.

26. Kobayashi Y, Suzuki M, Satsu H, et al. (2000) Green tea polyphenols inhibit the sodium-dependent glucose transporter of intestinal epithelial cells by a competitive mechanism. J Agric Food Chem 48, 5618-5623.

27. Manzano S \& Williamson G (2010) Polyphenols and phenolic acids from strawberry and apple decrease glucose uptake and transport by human intestinal Caco-2 cells. Mol Nutr Food Res 54, 1773-1780.

28. Nyambe-Silavwe H, Villa-Rodriguez JA, Ifie I, et al. (2015) Inhibition of human $\alpha$-amylase by dietary polyphenols. J Funct Foods 19, 723-732.

29. Adisakwattana S, Charoenlertkul P \& Yibchok-anun S (2009) alpha-Glucosidase inhibitory activity of cyanidin-3-galactoside and synergistic effect with acarbose. I Enzyme Inhib Med Chem 24, 65-69.

30. Singleton VL, Orthofer R \& Lamuela-Raventos RM (1999) Analysis of total phenols and other oxidation substrates and antioxidants by means of Folin-Ciocalteu reagent. In Methods in Enzymology, vol. 299, pp. 152-178 [L Packer, editor]. San Diego, CA: Elsevier Academic Press Inc.

31. AOAC International (2005) Official Methods of Analysis of AOAC International. Gaithersburg, MD: Association of Official Analytical Chemists.

32. Neveu V, Perez-Jimenez J, Vos F, et al. (2010) PhenolExplorer: an online comprehensive database on polyphenol contents in foods. Database 2010, bap024.

33. Anderson JW, Baird P, Davis RH, et al. (2009) Health benefits of dietary fiber. Nutr Rev 67, 188-205.

34. Weickert MO \& Pfeiffer AF (2008) Metabolic effects of dietary fiber consumption and prevention of diabetes. J Nutr $\mathbf{1 3 8}$, 439-442.

35. Willett W, Manson J \& Liu S (2002) Glycemic index, glycemic load, and risk of type 2 diabetes. Am J Clin Nutr $\mathbf{7 6}$, 274S-280S

36. Bryans JA, Judd PA \& Ellis PR (2007) The effect of consuming instant black tea on postprandial plasma glucose and insulin concentrations in healthy humans. I Am Coll Nutr 26, 471-477.

37. Chai Y, Wang M \& Zhang G (2013) Interaction between amylose and tea polyphenols modulates the postprandial glycemic response to high-amylose maize starch. J Agric Food Chem 61, 8608-8615.

38. Clegg ME, Pratt M, Meade CM, et al. (2011) The addition of raspberries and blueberries to a starch-based food does not alter the glycaemic response. Br J Nutr 106, 335-338.

39. Coe SA, Clegg M, Armengol M, et al. (2013) The polyphenolrich baobab fruit (Adansonia digitata L.) reduces starch digestion and glycemic response in humans. Nutr Res 33, 888-896.

40. Hlebowicz J, Darwiche G, Bjorgell O, et al. (2007) Effect of cinnamon on postprandial blood glucose, gastric emptying, and satiety in healthy subjects. Am J Clin Nutr 85 , $1552-1556$

41. Johnston KL, Clifford MN \& Morgan LM (2002) Possible role for apple juice phenolic, compounds in the acute modification of glucose tolerance and gastrointestinal hormone secretion in humans. J Sci Food Agric 82, 1800-1805. 
42. Johnston KL, Clifford MN \& Morgan LM (2003) Coffee acutely modifies gastrointestinal hormone secretion and glucose tolerance in humans: glycemic effects of chlorogenic acid and caffeine. Am J Clin Nutr 78, 728-733.

43. Josic J, Olsson AT, Wickenberg J, et al. (2010) Does green tea affect postprandial glucose, insulin and satiety in healthy subjects: a randomized controlled trial. Nutr J 9, 63.

44. Linderborg KM, Jarvinen R, Lehtonen HM, et al. (2012) The fiber and/or polyphenols present in lingonberries null the glycemic effect of the sugars present in the berries when consumed together with added glucose in healthy human volunteers. Nutr Res 32, 471-478.

45. Makarova E, Gornas P, Konrade I, et al. (2015) Acute anti-hyperglycaemic effects of an unripe apple preparation containing phlorizin in healthy volunteers: a preliminary study. J Sci Food Agric 95, 560-568.
46. Schulze C, Bangert A, Kottra G, et al. (2014) Inhibition of the intestinal sodium-coupled glucose transporter 1 (SGLT1) by extracts and polyphenols from apple reduces postprandial blood glucose levels in mice and humans. Mol Nutr Food Res 58, 1795-1808.

47. Torronen R, Sarkkinen E, Niskanen T, et al. (2012) Postprandial glucose, insulin and glucagon-like peptide 1 responses to sucrose ingested with berries in healthy subjects. Br J Nutr 107, 1445-1451.

48. Tsujita $\mathrm{T} \&$ Takaku $\mathrm{T}$ (2008) Mechanism of the inhibitory action of chestnut astringent skin extract on carbohydrate absorption. J Nutr Sci Vitaminol 54, 416-421.

49. Tsujita T, Takaku T \& Suzuki T (2008) Chestnut astringent skin extract, an alpha-amylase inhibitor, retards carbohydrate absorption in rats and humans. J Nutr Sci Vitaminol $\mathbf{5 4}$, $82-88$. 